\title{
NATURAL CONVECTION COOLING OF A HOT VERTICAL WALL WET BY A FALLING LIQUID FILM
}

\author{
Gaetano Aiello and Michele Ciofalo \\ Dipartimento di Ingegneria Nucleare, Università degli Studi di Palermo \\ Viale delle Scienze ed.6, 90128 Palermo, Italy.Email: ciofalo@din.din.unipa.it
}

\begin{abstract}
The system studied is a plane channel delimited by two vertical walls, one of which is imposed an arbitrary temperature profile and may be partially or totally wet by a liquid film, while the other is adiabatic. Air from the environment flows along the channel, driven by buoyancy forces. Its mass flow rate depends on the hydraulic resistances and on the distribution of temperature and humidity (hence, density) along the channel, which, in turn, depends on the heat and mass transfer between hot wall and humid air. Due to evaporative or boiling mass transfer, the liquid film, if present, shrinks as it descends along the hot wall, and may be completely dried out at some height.

A simplified computational model of the above system was developed and applied to the prediction of relevant quantities, such as the total energy subtracted to the hot wall, for a range of conditions (hot wall temperature and its distribution; film flow rate; ambient air temperature and humidity; channel height and thickness; localized hydraulic resistance).
\end{abstract}

\section{PROBLEM DESCRIPTION}

The cooling of a hot vertical wall by natural convection of air, with or without the assistance of a falling liquid film, is a fundamental heat transfer problem which is also encountered in nuclear reactor safety, for example in the containment cooling following a loss of coolant accident in AP600/AP1000 facilities (Kang and Park, 2001).

The system studied here is schematically shown in Fig. 1. A channel of height $H$ and width $s$ is delimited by two vertical walls. One wall is imposed an arbitrary temperature profile $T_{w}(x)$ and may or not be wet by a liquid film, falling along it with an inlet flow rate $\Gamma_{l, H}$ and an inlet temperature $T_{l, H}$. The opposite wall is adiabatic. Air from the environment, at temperature $T_{0}$ and humidity $m_{0}$, flows along the channel, driven by buoyancy forces. The inlet air mass flow rate $\Gamma_{a, 0}$ depends on the distribution of air temperature $T_{a}$ and humidity $m$ (hence, density $\rho_{a}$ ) along the channel, which, in turn, depends on the heat and mass transfer between the hot wall (either dry or wet by a liquid film) and the humid air. Besides the distributed frictional resistances, the air flow encounters a localized hydraulic resistance $K$. The liquid film, if present, experiences a progressive reduction of its flow rate and thickness as it descends along the hot wall, due to evaporative or boiling mass transfer to the humid air flow, and may be completely dried out at a height $x^{*}$. Steady state conditions are assumed.

Here, $\Gamma_{l}$ and $\Gamma_{a}$ denote flow rates per unit length in the direction orthogonal to the plane of the figure. The overall geometry can be thought of as an annulus of negligible curvature, as in the AP600 containment cooling configuration. 


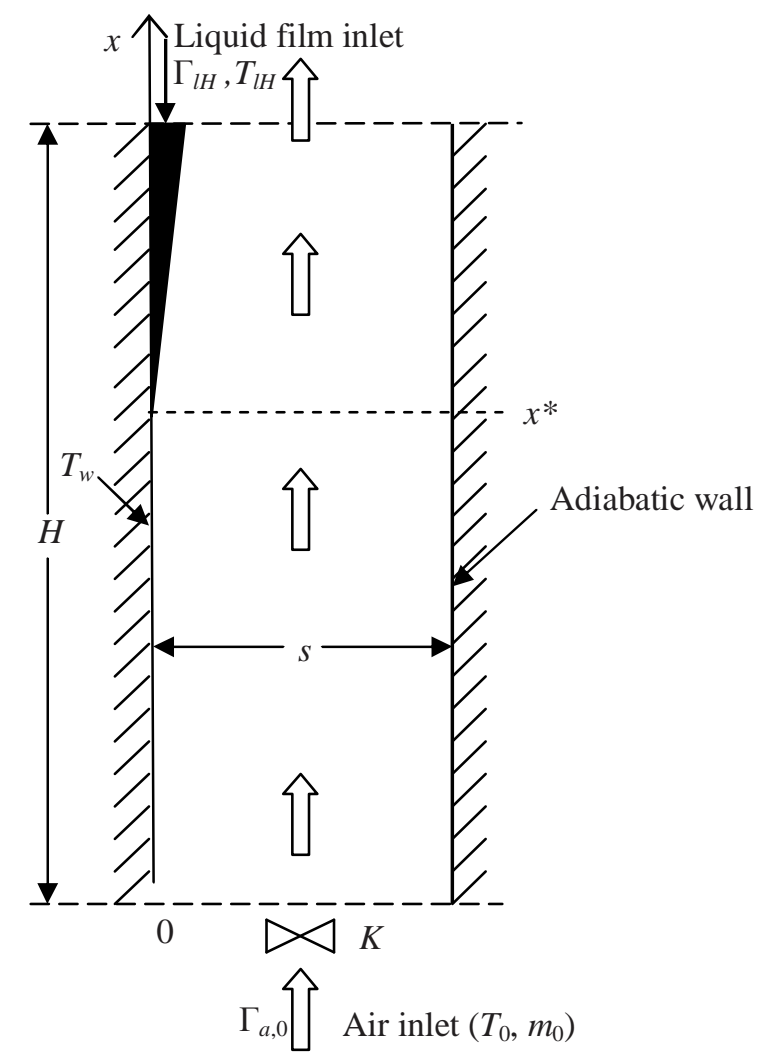

Figure 1. Schematic of the physical system.

The localized hydraulic resistance may be thought of as representative of the overall ambient air path upstream of the vertical channel proper. Further modelling assumptions are:

- The thermal resistance of the liquid film is negligible with respect to the film-air thermal resistance; therefore, the film temperature is equal to the lower between $T_{w}$ and $T_{\text {sat }}$.

- There is no flooding, which implies limits to the air velocity (Bankoff and Lee, 1986).

- Boiling, if present $\left(T_{w}>T_{s a t}\right.$ ), is nucleate (which implied $T_{w}<T_{D N B}$ ).

- The air flow is perfectly mixed (i.e., air temperature and humidity are functions of $x$ only).

- The system pressure is uniform and equal to the ambient pressure $p_{0}$.

- Although the air flows driven by buoyancy forces, forced-convection correlations can be used for friction and heat transfer due to the high aspect ratio of the channel $(H \gg s)$.

\section{GOVERNING EQUATIONS}

With reference to Fig. 1, the conservation equations which govern the phenomenon are:

- Humid air mass balance (constant dry air flow, variable water component flow):

$$
\frac{d \Gamma_{a}}{d x}=\frac{d\left(m \Gamma_{a}\right)}{d x}=m^{\prime \prime}
$$

- Liquid film mass balance (if appropriate):

$$
\frac{d \Gamma_{l}}{d x}=m^{\prime \prime}
$$

- Humid air enthalpy balance: 


$$
\frac{d J_{a}}{d x}=q^{\prime \prime}
$$

- Humid air flow momentum balance, including buoyancy, pressure losses and inlet-outlet momentum change:

$$
\Delta p_{\text {buo }}-\left|\Delta p_{\text {loss }}\right|=\frac{\Gamma_{a, H}^{2}}{\rho_{a, H} s^{2}}-\frac{\Gamma_{a, 0}^{2}}{\rho_{a, 0} s^{2}}
$$

in which the driving buoyancy term and the overall loss term are given by:

$$
\begin{gathered}
\Delta p_{\text {buo }}=\int_{0}^{H}\left(\rho_{a, 0}-\rho_{a}\right) g d x \\
\left|\Delta p_{\text {loss }}\right|=\int_{0}^{H} f \frac{1}{2 D_{e q} \rho_{a}}\left(\frac{\Gamma_{a}}{s}\right)^{2} d x+K \frac{1}{2 \rho_{a, 0}}\left(\frac{\Gamma_{a, 0}}{s}\right)^{2}
\end{gathered}
$$

respectively, $D_{e q}=2 s$ being the hydraulic diameter of the channel.

As regards heat and mass transfer correlations (kinetic equations), the total heat flux from hot wall to humid air flow is expressed in the most general case as:

$$
q^{\prime \prime}=q_{\text {conv }}^{\prime \prime}+q_{\text {evap }}^{\prime \prime}+q_{\text {boil }}^{\prime \prime}
$$

- The convective heat flux $q_{\text {conv }}^{\prime \prime}$ is always present and is computed as:

$$
q_{\text {conv }}^{\prime \prime}=h\left(T_{w}-T_{a}\right)
$$

in which the convective coefficient $h$ is computed by the Dittus-Bölter correlation:

$$
\frac{h D_{e q}}{\lambda_{a}}=0.023\left(\frac{\Gamma_{a} D_{e q}}{\mu_{a} s}\right)^{0.8}\left(\frac{c_{p a} \mu_{a}}{\lambda_{a}}\right)^{0.4}
$$

- Evaporative heat and mass transfer exist only in the presence of the liquid film. The evaporative mass flux is computed as:

$$
m_{\text {evap }}^{\prime \prime}=h_{m}\left(m_{\delta}-m_{\text {eff }}\right)
$$

in which $m_{\delta}=m_{s a t}\left(T_{w}\right)$ is the humidity at the film surface and $m_{\text {eff }}=\min \left[m, m_{s a t}\left(T_{a}\right)\right]$. For the mass transfer coefficient $h_{m}$ the Sherwood analogy is used (Song et al., 2003):

$$
h_{m} \cong \frac{h}{c_{p a}}
$$

where, in the absence of reliable correlations, the specific heat of humid air is approximated by that of dry air. The evaporative film - air heat flux is then computed as:

$$
q_{\text {evap }}^{\prime \prime}=m_{\text {evap }}^{\prime \prime} j_{g}
$$

in which $j_{\mathrm{g}}$ is the enthalpy of saturated vapour at the wall (and liquid film) temperature. 
- Boiling heat and mass transfer are present only for $T_{w}>T_{\text {sat }}$ (and, of course, in the presence of the liquid film). The boiling mass flux is computed from the Jens and Lottes correlation:

$$
m_{b o i l}^{\prime \prime}=\frac{f(p)}{j_{f g}}\left(T_{w}-T_{s a t}\right)^{4}
$$

(Collier and Thome, 1996), in which $f(p)=2.73$ (SI units) at atmospheric pressure. The boiling film - air heat flux is then computed, by analogy with the evaporative one, as:

$$
q_{b o i l}^{\prime \prime}=m_{b o i l}^{\prime \prime} j_{g}
$$

Finally, as regards the relevant thermodynamic relations, the humidity $m$, defined as the water/mixture mass ratio, is related to the volume, molar and pressure ratio $\psi$ by:

$$
\frac{1}{\psi}=\left(1-\frac{M_{l}}{M_{a}}\right)+\frac{1}{m} \frac{M_{l}}{M_{a}}
$$

in which $M_{l}=18.016 \cdot 10^{-3} \mathrm{~kg} / \mathrm{mol}$ and $M_{a}=28.978 \cdot 10^{-3} \mathrm{~kg} / \mathrm{mol}$ are the molar weights of water and standard air, respectively (Perry and Green, 1984). The saturation humidity $m_{s a t}$ is attained when the partial pressure of water vapour equals the saturation pressure of water at the humid air temperature $T_{a}$, i.e. when $\psi=p_{s a t}\left(T_{a}\right) / p_{0}$; equation (15) thus yields:

$$
m_{s a t}\left(T_{a}\right)=\frac{M_{l}}{M_{a}}\left[\frac{p_{0}}{p_{s a t}\left(T_{a}\right)}-\left(1-\frac{M_{l}}{M_{a}}\right)\right]^{-1}
$$

Approximate correlations, based on the "UK Steam Tables in SI Units" (1970) and valid in the range $0-150^{\circ} \mathrm{C}$, were used in the calculations to compute the saturation pressure $p_{\text {sat }}(T)$, the saturation temperature $T_{s a t}(p)$ and the latent heat of vaporization of water $j_{f g}(T)$. The enthalpy of saturated vapour was then computed as:

$$
j_{g}(T)=c_{p l} \cdot\left(T-T_{r e f}\right)+j_{f g}(T)
$$

with $c_{p l}=4186 \mathrm{~J} /(\mathrm{kg} \cdot \mathrm{K})$ and $T_{r e f}=273.15 \mathrm{~K}\left(0^{\circ} \mathrm{C}\right)$. The enthalpy of overheated vapor was computed as:

$$
j_{v}\left(T, p_{v}\right)=j_{g}\left[T_{s a t}\left(p_{v}\right)\right]+c_{p v}\left[T-T_{s a t}\left(p_{v}\right)\right]
$$

with $c_{p v}=1870 \mathrm{~J} /(\mathrm{kg} \cdot \mathrm{K})$. The total enthalpy of humid air was computed by the formulae:

$$
\begin{aligned}
& j_{a}=(1-m) c_{p a}\left(T_{a}-T_{r e f}\right)+m j_{g}\left[T_{s a t}\left(p_{v}\right)\right]+m c_{p v}\left[T_{a}-T_{s a t}\left(p_{v}\right)\right] \\
& j_{a}=(1-m) c_{p a}\left(T_{a}-T_{r e f}\right)+m_{s a t} j_{g}\left(T_{a}\right)+\left(m-m_{s a t}\right) c_{p l}\left(T_{a}-T_{r e f}\right)
\end{aligned}
$$

valid for undersaturated air $\left(m \leq m_{s a t}\right)$ and oversaturated air $\left(m>m_{s a t}\right)$, respectively. Similarly, the density of humid air was computed by the formulae:

$$
\begin{gathered}
\rho_{a}(m, T)=10^{-3} \frac{p_{0}}{R T}\left[\frac{1-m}{M_{a}}+\frac{m}{M_{l}}\right]^{-1} \\
\rho_{a}(m, T)=10^{-3} \frac{p_{0}}{R T}\left[\left(\frac{1-m}{M_{a}}+\frac{m_{\text {sat }}}{M_{l}}\right)+\frac{m-m_{\text {sat }}}{\rho_{l}}\right]^{-1}
\end{gathered}
$$


valid for undersaturated air $\left(m \leq m_{s a t}\right)$ and oversaturated air $\left(m>m_{\text {sat }}\right)$, respectively.

The energy balance of the system considered is schematically shown in Fig. 2 for the general case in which a residual liquid water film flow $\Gamma_{l, 0}$ reaches the base of the wall. It should be observed that the power $W_{a}$ absorbed by the humid air flow:

$$
W_{a}=\Gamma_{a, H} j_{a, H}-\Gamma_{a, 0} j_{a, 0}
$$

differs, in general, from the power $W_{w}$ subtracted to the hot wall and satisfies:

$$
W_{w}=W_{a}-\left(\Gamma_{l, H} j_{l, H}-\Gamma_{l, 0} j_{l, 0}\right)
$$

where $j_{l, H}$ and $j_{l, O}$ are the enthalpies of the liquid water film at the top and bottom of the wall.

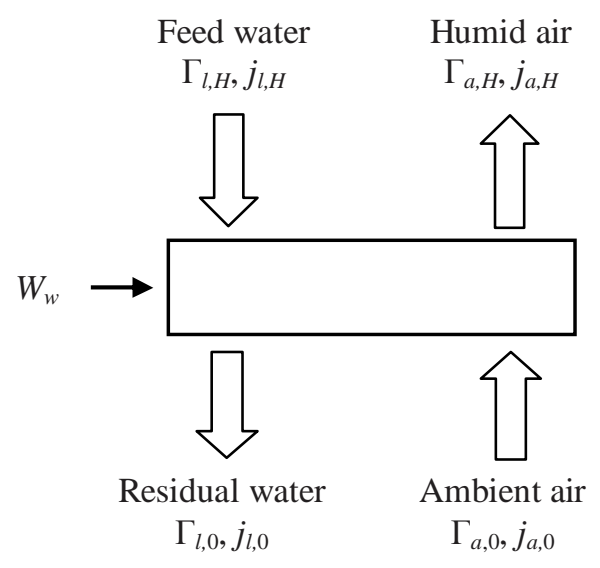

Figure 2. Energy balance of the physical system.

The above equations were discretized by the finite-volume method and numerically solved by a purpose written Fortran code. Details are given by Aiello (2008). In principle, it would be possible to choose a value of the inlet film flow rate $\Gamma_{l, H}$ and compute the dryout location $x^{*}$ as part of the solution. However, an iterative approach would then be necessary since the dry/wet status of the wall at a generic height would not be known a priori. It was found easier to loop on the film dryout height $x^{*}$ (from 0 to $H$ ) and compute for each value of $x^{*}$ both the film and the air flow rates. A simple iterative procedure was still necessary to compute the air flow rate satisfying the balance between buoyancy forces, pressure losses and inlet-outlet momentum change (equations 4 through 6).

\section{RESULTS AND DISCUSSION}

In the following, the ambient conditions are $T_{0}=10^{\circ} \mathrm{C}, m_{0}=3 \times 10^{-3}$, atmospheric pressure. Figure 3 reports the inlet air flow rate $\Gamma_{a, 0}$ as a function of the wall temperature $T_{w}$ (assumed uniform) for $s=0.2 \mathrm{~m}, K=5$ and three values of the wall height $H$ in the two limiting cases $x^{*}=H$ (dry wall) and $x^{*}=0$ (wall entirely wet by a liquid film). It can be observed that the air flow rate generally increases with the wall temperature and is much higher for a wet wall than for a dry wall. Only when the wall temperature exceeds $100^{\circ} \mathrm{C}$, giving rise to nucleate boiling of the liquid film, the inlet flow rate decreases with $T_{w}$; however, the outlet flow rate (not shown here) monotonically increases with $T_{w}$ in all cases as high values of $T_{w}$ are associated with very high outlet humidity, well above saturation (mist). 


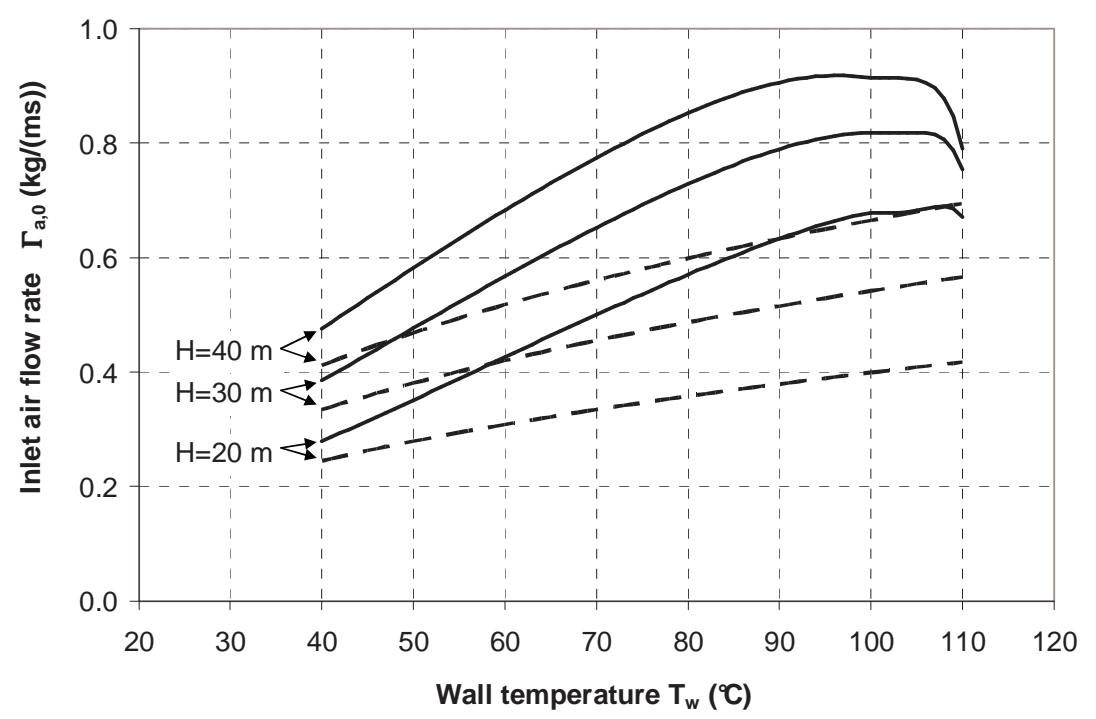

Figure 3. Inlet air flow rate as a function of $T_{w}$ for $s=0.2 \mathrm{~m}, K=5$ and three values of $H$. Broken lines: dry wall $\left(x^{*}=H\right)$; solid lines: totally wet wall $\left(x^{*}=0\right)$.

Figure 4 reports the total power $W_{w}$ subtracted to the wall under the same conditions. The use of a $\log$ scale should be noticed. In the presence of a liquid film $W_{w}$ is much larger than for a dry wall, and increases much more steeply with $T_{w}$ (or, better, with $T_{w}-T_{0}$ ). Interestingly, the increase of $W_{w}$ with $T_{w}$ stops for $T_{w}=T_{\text {sat }}$, when the film surface humidity $m_{\delta}=m_{s a t}\left(T_{w}\right)$ in equation (10) for the evaporative mass (and heat) flux attains its maximum value of 1 . For further increases of $T_{w}$ beyond $T_{\text {sat }}$ the evaporative flux remains unchanged, while a boiling mass/heat flux appears which, however, remains small until $T_{w}-T_{\text {sat }}$ exceeds a few ${ }^{\circ} \mathrm{C}$.

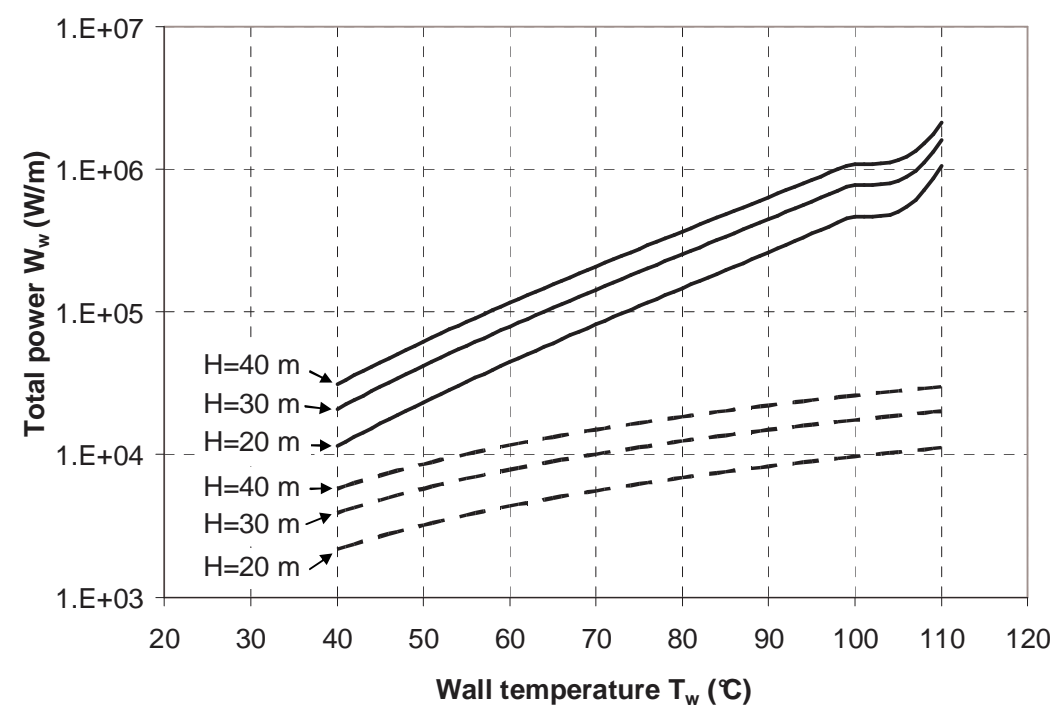

Figure 4. Total power subtracted to the wall as a function of $T_{w}$ for $s=0.2 \mathrm{~m}, K=5$ and three values of $H$. Broken lines: dry wall $\left(x^{*}=H\right)$; solid lines: totally wet wall $\left(x^{*}=0\right)$.

Fig. 5 reports the total power $W_{w}$ subtracted to the wall as a function of the channel width $s$ for $T_{w}=100^{\circ} \mathrm{C}$ (uniform), $H=30 \mathrm{~m}$, three values of $K$ and $x^{*}=0$ (wet wall) or $H$ (dry wall). An optimum width, yielding maximum heat transfer, exists in all cases; it decreases with $K$ and (for the present case) is $0.13 \sim 0.14 \mathrm{~m}$ for a dry wall and $0.18 \sim 0.26 \mathrm{~m}$ for a totally wet wall. 


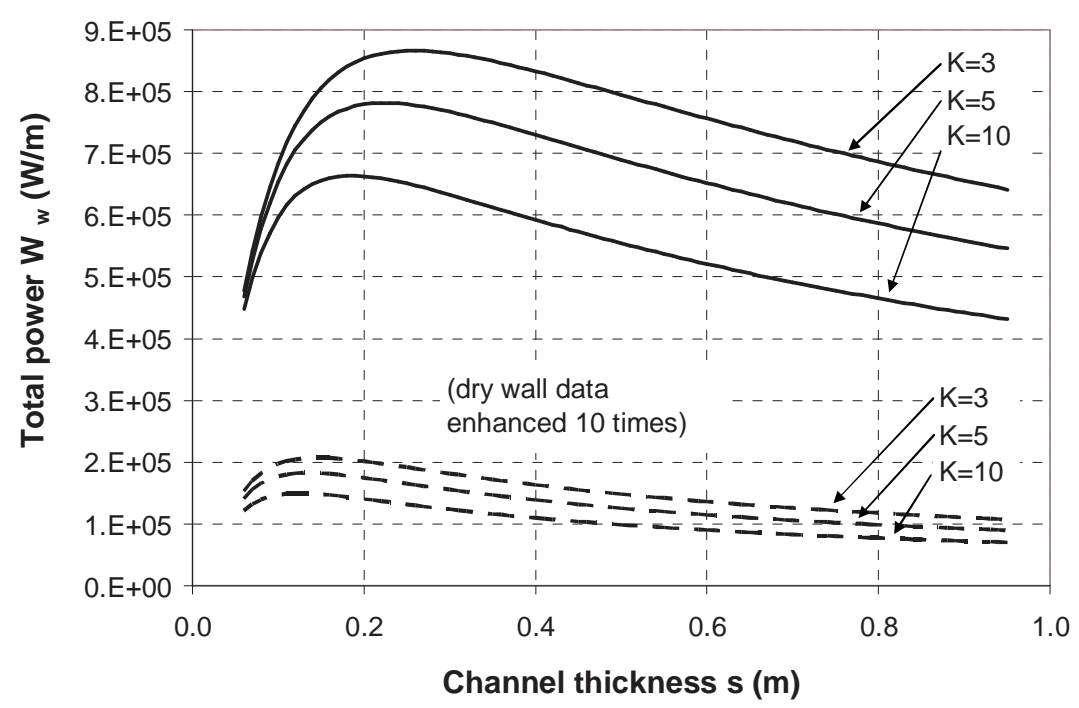

Figure 5. Total power subtracted to the wall as a function of $s$ for $T_{\mathrm{w}}=100^{\circ} \mathrm{C}, H=30 \mathrm{~m}$ and three values of $K$. Broken lines: dry wall $\left(x^{*}=H\right)$; solid lines: totally wet wall $\left(x^{*}=0\right)$.

Fig. 6 reports the total power $W_{w}$ subtracted to the wall as a function of the film inlet flow rate $\Gamma_{l, H}$ for $H=30 \mathrm{~m}, s=0.2 \mathrm{~m}, K=5$ and different values of $T_{w}$. For each $T_{w}, W_{w}$ starts from a small value $W_{W}(0)$ corresponding to a dry wall $\left(x^{*}=H\right)$. As $\Gamma_{l, H}$ increases, the wet length $H$ - $x^{*}$ grows and $W_{w}$ increases linearly with $\Gamma_{l, H}$ up to a value $\Gamma_{l, H}{ }^{*}$, corresponding to the condition $x^{*}=0$ (totally wet wall). For larger $\Gamma_{l, H}$, an increasing fraction of the film flow rate reaches the base of the wall in the liquid state and $W_{w}$ grows more slowly, because of the sensibile heat required to warm the liquid from its inlet temperature $T_{l, H}$ to the thermal equilibrium temperature $T_{w}$, whereas the power $W_{\mathrm{a}}$ transmitted to the air flow remains unchanged (Fig. 2).

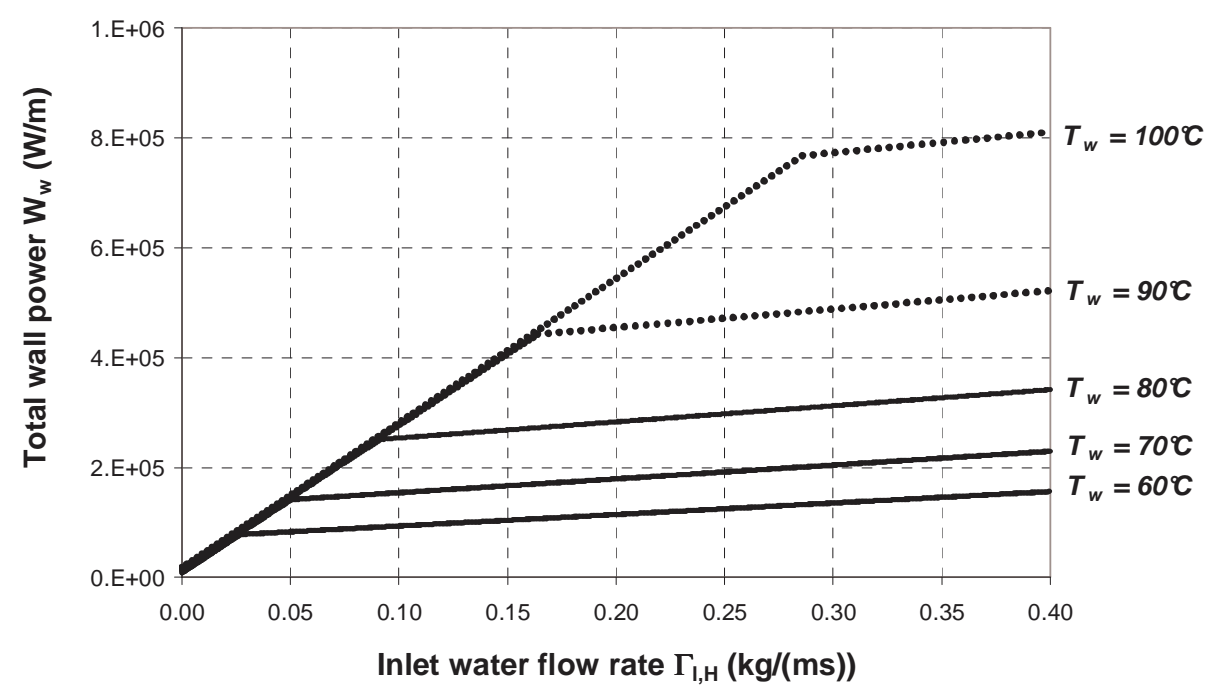

Figure 6. Total power subtracted to the wall as a function of the film flow rate for $H=30 \mathrm{~m}, s=0.2$ $\mathrm{m}, K=5$ and different values of $T_{w}$.

Now, assume that the feed water for the liquid film is drawn from a reservoir of finite mass $M_{l}$ (per unit perimeter). The mass flow rate $\Gamma_{l, H}-$ supposed constant - can only be provided up to a time $t_{w e t}=M_{l} /\left(\Gamma_{l, H}\right)$. The power subtracted to the wall will be $W_{w}\left(\Gamma_{l, H}\right)$ for $0 \leq t<t_{w e t}$ and $W_{w}(0)$ for $t>t_{\text {wet }}$. On the basis of results like those in Fig. 6, and assuming that $T_{\mathrm{w}}$ remains constant throughout the cooling process, the total thermal energy $E_{T}\left(t, t_{w e t}\right)$ subtracted to the wall up to 
time $t$ can be computed for different values of $t_{\text {wet }}$, or "cooling strategies"(Fig. 7). For each $t_{w e t}$, the corresponding curve in Fig. 7 exhibits an abrupt change of slope at $t=t_{\text {wet }}$, associated with the transition from wet to dry cooling. The solid curve is for $t_{w e t}$ equal to $t_{w e t}{ }^{*}=M_{l} /\left(\Gamma_{l, H}{ }^{*}\right)$, $\Gamma_{l, H}{ }^{*}$ being the minimum flow rate for which the wall is totally covered by the liquid film $\left(x^{*}=0\right)$. For $t_{\text {wet }}>t_{\text {wet }}{ }^{*}\left(\Gamma_{l, H}<\Gamma_{l, H}{ }^{*}\right)$ the integrated thermal energy subtracted to the wall is at any time less or equal to that corresponding to $t_{w e t}=t_{w e t}{ }^{*}$, whereas for $t_{w e t}<t_{w e t}{ }^{*}\left(\Gamma_{l, H}>\Gamma_{l, H}{ }^{*}\right)$ it exceeds slightly $E_{T}\left(t, t_{\text {wet }}{ }^{*}\right)$ for $t<t_{\text {wet }}$, but remains lower for any larger time. Thus, in a well defined sense, the "cooling strategy" characterized by the choice $t_{w e t}=t_{w e t}{ }^{*}$ is optimal.

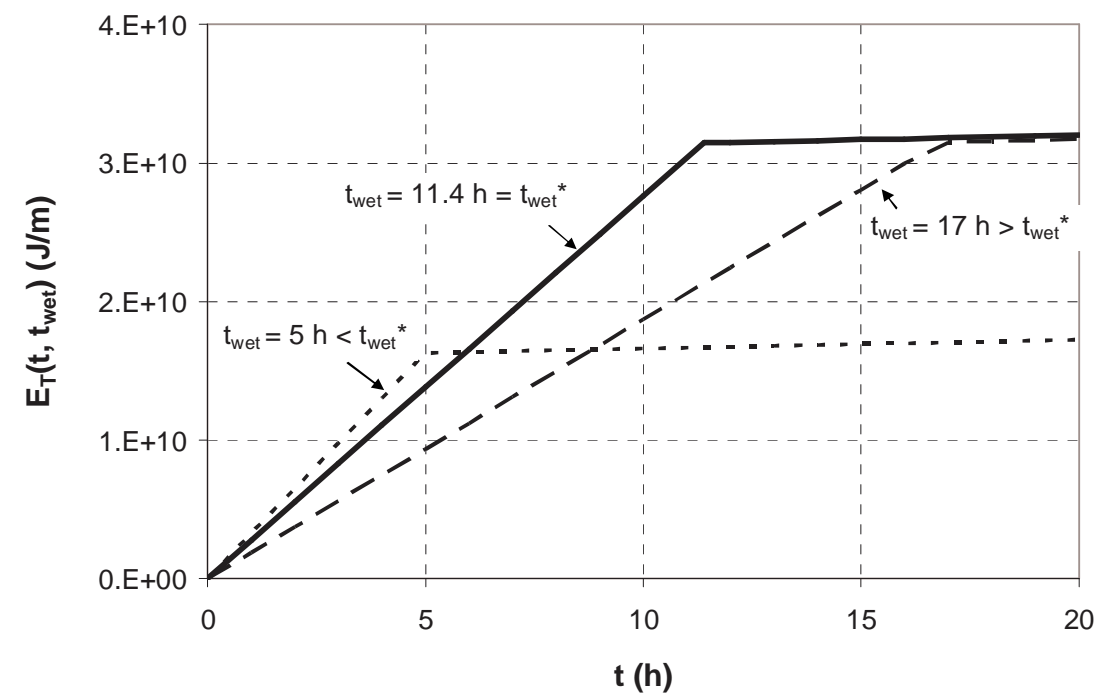

Figure 7. Total thermal energy subtracted from the wall as a function of time for $T_{w}=100^{\circ} \mathrm{C}$, $H=30 \mathrm{~m}, K=5$ and different "cooling strategies".

\section{Conclusions}

A model was developed for the free convection cooling of a hot vertical wall, either dry or wet by a liquid water film. It was used to predict the dependence of relevant quantities, e.g. air flow rate and thermal power subtracted to the wall, on system's parameters such as wall temperature, channel height and width, localized hydraulic resistance and water supply rate. Notably, the existence of an optimum channel width was predicted. In the case of a finite water reservoir and of a constant wall temperature, the existence of an optimum "cooling strategy" was demonstrated, consisting of supplying water at the minimum flow rate which guarantees a complete coverage of the wall by the liquid film.

\section{REFERENCES}

Aiello, G., 2008, Master's Thesis in Nuclear Engineering, University of Palermo, Italy.

Bankoff, S.G. and S.C. Lee, 1986, in Multiphase Science and Technology, Eds. G.F. Hewitt, J.M. Delhaye and N. Zuber, Hemisphere - McGraw-Hill, New York.

Collier, J.G. and J.R. Thome, 1996, Convective Boiling and Condensation, $3^{\text {rd }}$ edition, Clarendon Press, Oxford.

Kang, Y.M. and G.C. Park, 2001, Nucl. Eng. and Design 204, 347-359.

Perry, R.H. and D.W. Green, 1984, Perry's Chemical Engineers' Handbook, McGraw-Hill, Singapore.

Song, C.H., D.-Y. Lee and S.T. Ro, 2003, Int. J. Heat Mass Transfer 46, 1241-1249.

U.K. Committee on the Properties of Steam, 1970, UK Steam Tables in SI Units 1970, Edward Arnold Publishers, London. 